\title{
Periodic Solutions of Second-Order Difference Problem with Potential Indefinite in Sign
}

\author{
Honghua Bin \\ School of Science, Jimei University, Xiamen 361021, China \\ Correspondence should be addressed to Honghua Bin; hhbin@jmu.edu.cn
}

Received 19 January 2013; Accepted 24 February 2013

Academic Editor: Zhenkun Huang

Copyright ( 2013 Honghua Bin. This is an open access article distributed under the Creative Commons Attribution License, which permits unrestricted use, distribution, and reproduction in any medium, provided the original work is properly cited.

We investigate the periodic solutions of second-order difference problem with potential indefinite in sign. We consider the compactness condition of variational functional and local linking at 0 by introducing new number $\lambda_{*}$. By using Morse theory, we obtain some new results concerning the existence of nontrivial periodic solution.

\section{Introduction}

We consider the second-order discrete Hamiltonian systems

$$
\Delta^{2} x_{n-1}+W^{\prime}\left(n, x_{n}\right)=0, \quad x_{n+T}=x_{n},
$$

where $T \geq 2$ is a given integer, $n \in \mathbb{Z}, x_{n} \in \mathbb{R}^{N}, \Delta x_{n}=$ $x_{n+1}-x_{n}, \Delta^{2} x_{n}=\Delta\left(\Delta x_{n}\right), W^{\prime}$ stands for the gradient of $W$ with respect to the second variable. $W \in C^{2}\left(\mathbb{Z} \times \mathbb{R}^{N}, \mathbb{R}\right)$ is $T$-periodic in the first variable and has the form $W(n, x)=$ $(1 / 2) a|x|^{2}+H(n, x)$, where $a=4 \sin ^{2}(m \pi / T)$ for some $m \in$ $Z[0, r], r=[T / 2],[\cdot]$ stands for the greatest-integer function. For integers $a \leq b$, the discrete interval $\{a, a+1, \ldots, b\}$ is denoted by $Z[a, b]$.

In this paper we consider that $H$ is sign changing, that is,

$$
\begin{aligned}
H(n, x) & =b(n)\left(\frac{1}{s}|x|^{s}+\bar{G}_{s}(n, x)\right) \\
& \triangleq \frac{1}{s} b(n)|x|^{s}+G_{s}(n, x),
\end{aligned}
$$

$\left.\left.\Omega_{+}=\{n \in Z[1, T] \mid b(n)>0)\right\}, \Omega_{-}=\{n \in Z[1, T] \mid b(n)<0)\right\}$ are two nonempty subsets of $Z[1, T]$, where $s>1, b(\cdot)$ is a $T$ periodic real function, $G_{s} \in C^{1}\left(\mathbb{Z} \times \mathbb{R}^{N}, \mathbb{R}\right)$, and $G_{s}(n, 0)=0$.
Consider the second-order Hamiltonian system

$$
\begin{gathered}
\ddot{x}(t)+W^{\prime}(t, x)=0, \quad x(0)=x(T), \\
\dot{x}(0)=\dot{x}(T),
\end{gathered}
$$

where $W \in C^{2}\left(\mathbb{R} \times \mathbb{R}^{N}, \mathbb{R}\right)$ is $T$-periodic in $t, W(t, x)=$ $(1 / 2)(A(t) x, x)+H(t, x)$. Here $A(\cdot)$ is a continuous, $T$ periodic matrix-value function.

Systems (1) and (3) have been investigated by many authors using various methods, see [1-5]. The dynamical behavior of differential and difference equations was studied by using various methods, and many interesting results have obtained, see [6-10] and references therein. The critical point theory [11-14] is a useful tool to investigate differential equations. Morse theory [15-19] has also been used to solve the asymptotically linear problem. By minimax methods in critical point theory, Tang and $\mathrm{Wu}[4]$, Antonacci $[20,21]$ considered the problem (3) with potential indefinite in sign, where $H$ is superquadratic at zero and infinity. By using Morse theory, Zou and $\mathrm{Li}[10]$ study the existence of $T$-periodic solution of (3), where $H$ is asymptotically superquadratic and sign changing. Moroz [19] studies system (3) where $H$ is asymptotically subquadratic and sign changing. Motivated by $[5,10,19]$, we investigate periodic solutions for asymptotically superquadratic or subquadratic discrete system (1).

By expression of $H(n, x)$, system (1) possesses a trivial solution $x=0$. Here we are interested in finding the nonzero $T$-periodic solution of (1), and we divide the problem into two cases: $s>2$ and $1<s<2$. For $s=2$, one can refer to [22]. 
Case 1 (asymptotically superquadratic case: $s>2$ ). In this case, we replace $p$ with $s$ in (2). Letting $g_{p}(n, x)=G_{p}^{\prime}(n, x)$, we rewrite (1) as

$$
\begin{gathered}
\Delta^{2} x_{n-1}+a x_{n}+b(n)\left|x_{n}\right|^{p-2} x_{n}+g_{p}\left(n, x_{n}\right)=0, \\
x_{n+T}=x_{n} .
\end{gathered}
$$

Furthermore, for all $(n, x) \in \mathbb{Z} \times \mathbb{R}^{N}$, we assume that $g_{p}$ satisfies

(A1) $g_{p}(n, x)=o(|x|)$ as $|x| \rightarrow \infty$ uniformly in $n$,

(A2) $g_{p}(n, x)=o\left(|x|^{p-1}\right)$ as $|x| \rightarrow 0$ uniformly in $n$.

Case 2 (asymptotically subquadratic case: $1<s<2$ ). Here we replace $q$ with $s$ in (2). Letting $g_{q}(n, x)=G_{q}^{\prime}(n, x)$, we rewrite (1) as

$$
\begin{gathered}
\Delta^{2} x_{n-1}+a x_{n}+b(n)\left|x_{n}\right|^{q-2} x_{n}+g_{q}\left(n, x_{n}\right)=0, \\
x_{n+T}=x_{n} .
\end{gathered}
$$

For all $(n, x) \in \mathbb{Z} \times \mathbb{R}^{N}$, we assume that $g_{q}$ satisfies

(B1) $g_{q}(n, x)=o\left(|x|^{q-1}\right)$ as $|x| \rightarrow \infty$ uniformly in $n$,

(B2) $g_{q}(n, x)=o(|x|)$ as $|x| \rightarrow 0$ uniformly in $n$.

Before stating the main results, we introduce space $E_{T}=$ $\left\{x=\left\{x_{n}\right\} \in S \mid x_{n+T}=x_{n}, n \in \mathbb{Z}\right\}$, where $S=\{x=$ $\left.\left\{x_{n}\right\} \mid x_{n} \in \mathbb{R}^{N}, n \in \mathbb{Z}\right\}$. For any $x, y \in S, a, b \in \mathbb{R}$, we define $a x+b y=\left\{a x_{n}+b y_{n}\right\}_{n \in \mathbb{Z}}$. Then $S$ is a linear space. Let $\langle x, y\rangle_{E_{T}}=\sum_{n=1}^{T}\left(x_{n}, y_{n}\right),\|x\|_{E_{T}}=\left(\sum_{n=1}^{T}\left|x_{n}\right|^{2}\right)^{1 / 2}$, for all $x, y \in E_{T}$, where $(\cdot, \cdot)$ and $|\cdot|$ are the usual inner product and norm in $\mathbb{R}^{N}$, respectively. Obviously, $E_{T}$ is a Hilbert space with dimension NT and homeomorphism to $\mathbb{R}^{N T}$. For $r>1$, let $\|x\|_{r}=\left(\sum_{n=1}^{T}\left|x_{n}\right|^{r}\right)^{1 / r}, x \in E_{T}$. Moreover, for simplicity, we write $\langle x, y\rangle$ and $\|x\|$ instead of $\langle x, y\rangle_{E_{T}}$ and $\|x\|_{E_{T}}$, respectively.

Lemma 1. There exist positive numbers $a_{1}, a_{2}$, such that $a_{1} \|$ $x\left\|_{r} \leq\right\| x\left\|\leq a_{2}\right\| x \|_{r}$.

Inspired by [10,19], one introduces two numbers as follows:

$$
\begin{aligned}
& \lambda_{*}(p)=\inf _{\|x\|=1}\left\{\left.\|\Delta x\|^{2}\left|\sum_{n=1}^{T} b(n)\right| x_{n}\right|^{p}=0\right\}, \\
& \lambda_{*}(q)=\inf _{\|x\|=1}\left\{\left.\|\Delta x\|^{2}\left|\sum_{n=1}^{T} b(n)\right| x_{n}\right|^{q}=0\right\} .
\end{aligned}
$$

Theorem 2. If $a<\lambda_{*}(p)$, then (4) has a nonzero T-periodic solution.

Theorem 3. If $a<\lambda_{*}(q)$, then (5) has a nonzero T-periodic solution.

This paper is divided into four sections. Section 2 contains some preliminaries, and the proofs of Theorems 2 and 3 are given in Sections 3 and 4, respectively.

\section{Preliminaries}

2.1. Variational Functional and (PS) Condition. For seeking $T$-periodic solution of (1), we consider variational functional $J_{p}$ associated with (4) as $J_{p}(x)=(1 / 2) \sum_{n=1}^{T}\left|\Delta x_{n}\right|^{2}-$ $(1 / 2) a \sum_{n=1}^{T}\left|x_{n}\right|^{2}-1 / p \sum_{n=1}^{T} b(n)\left|x_{n}\right|^{p}-\sum_{n=1}^{T} G_{p}\left(n, x_{n}\right)$, that is

$$
\begin{aligned}
J_{p}(x)= & \frac{1}{2}\|\Delta x\|^{2}-\frac{1}{2} a\|x\|^{2}-\frac{1}{p} \sum_{n=1}^{T} b(n)\left|x_{n}\right|^{p} \\
& -\sum_{n=1}^{T} G_{p}\left(n, x_{n}\right), \quad x \in E_{T} .
\end{aligned}
$$

Moreover, $T$-periodic solution of (5) is associated with the critical point of functional

$$
\begin{aligned}
J_{q}(x)= & \frac{1}{2}\|\Delta x\|^{2}-\frac{1}{2} a\|x\|^{2}-\frac{1}{q} \sum_{n=1}^{T} b(n)\left|x_{n}\right|^{q} \\
& -\sum_{n=1}^{T} G_{q}\left(n, x_{n}\right), \quad x \in E_{T} .
\end{aligned}
$$

We say that a $C^{1}$-functional $\varphi$ on Hilbert space $X$ satisfies the Palais-Smale (PS) condition if every sequence $\left\{x^{(j)}\right\}$ in $X$, such that $\left\{\varphi\left(x^{(j)}\right)\right\}$, is bounded and $\varphi^{\prime}\left(x^{(j)}\right) \rightarrow 0$ as $j \rightarrow \infty$ contains a convergent subsequence.

Lemma 4. Functional $J_{p}$ satisfies (PS) condition if $a<\lambda_{*}(p)$.

Proof. Let $\left\{x^{(j)}\right\} \subset E_{T}$ be the (PS) sequence for functional $J_{p}$, such that $J_{p}\left(x^{(j)}\right)$ is bounded, and $J_{p}^{\prime}\left(x^{(j)}\right) \rightarrow 0$ as $j \rightarrow \infty$. Hence, for any $\varepsilon>0$, there exist $N_{\varepsilon}>0$ and constant $c_{1}>0$, such that

$$
\begin{array}{r}
\left|\left\langle J_{p}^{\prime}\left(x^{(j)}\right), x^{(j)}\right\rangle\right| \leq \varepsilon\left\|x^{(j)}\right\| \quad \text { for } j \geq N_{\varepsilon}, \\
\left|J_{p}\left(x^{(j)}\right)\right| \leq c_{1} .
\end{array}
$$

To prove that $J_{p}$ satisfies (PS) condition, it suffices to show that $\left\|x^{(j)}\right\|$ is bounded in $E_{T}$. Suppose not that there exists a subsequence $\left\{x^{\left(j_{k}\right)}\right\},\left\|x^{\left(j_{k}\right)}\right\| \rightarrow \infty$ as $k \rightarrow \infty$. For simplicity, we write as $\left\{x^{(j)}\right\}$ instead of $\left\{x^{\left(j_{k}\right)}\right\}$. Without loss of generality, we assume that there exists $k \in Z[1, T]$, such that

$$
\begin{gathered}
\left|x_{n}^{(j)}\right| \longrightarrow \infty \text { as } j \longrightarrow \infty \text { for } n \in Z[1, k], \\
x_{n}^{(j)} \text { are bounded for } n \in Z[k+1, T] .
\end{gathered}
$$

Therefore for all $n \in[1, T]$, by assumption (A1), there exists $c_{2}>0$ such that

$$
\begin{gathered}
\left|G_{p}\left(n, x_{n}^{(j)}\right)\right| \leq \varepsilon\left|x_{n}^{(j)}\right|^{2}+c_{2}, \\
\left|g_{p}\left(n, x_{n}^{(j)}\right)\right| \leq \varepsilon\left|x_{n}^{(j)}\right|+c_{2}
\end{gathered}
$$


for large $j$. By the previous argument, it follows that

$$
\begin{aligned}
\left|\sum_{n=1}^{T}\left(g_{p}\left(n, x_{n}^{(j)}\right), x_{n}^{(j)}\right)\right| & \leq \sum_{n=1}^{T}\left|g_{p}\left(n, x_{n}^{(j)}\right)\right|\left|x_{n}^{(j)}\right| \\
& \leq \varepsilon\left\|x^{(j)}\right\|^{2}+c_{2} T\left\|x^{(j)}\right\| .
\end{aligned}
$$

By (7), we have

$$
\begin{aligned}
p J_{p}\left(x^{(j)}\right)-\left\langle J_{p}^{\prime}\left(x^{(j)}\right), x^{(j)}\right\rangle \\
=\left(\frac{p}{2}-1\right)\left(\left\|\Delta x^{(j)}\right\|^{2}-a\left\|x^{(j)}\right\|^{2}\right)-p \sum_{n=1}^{T} G_{p}\left(n, x_{n}^{(j)}\right) \\
\quad+\sum_{n=1}^{T}\left(g_{p}\left(n, x_{n}^{(j)}\right), x_{n}^{(j)}\right) .
\end{aligned}
$$

In terms of (9) and (11), for large $j$, it follows that

$$
\begin{aligned}
& \left(\frac{p}{2}-1\right)\left(\left\|\Delta x^{(j)}\right\|^{2}-a\left\|x^{(j)}\right\|^{2}\right) \\
& \quad \leq p c_{1}+\varepsilon\left\|x^{(j)}\right\|+(p+1) \varepsilon\left\|x^{(j)}\right\|^{2}+p c_{2} T+c_{2} T\left\|x^{(j)}\right\| .
\end{aligned}
$$

Set $y_{n}^{(j)}=x_{n}^{(j)} /\left\|x^{(j)}\right\|$. Dividing by $\left\|x^{(j)}\right\|^{2}$ in the previous formula, it follows that

$$
\left\|\Delta y^{(j)}\right\|^{2} \leq a+\frac{2}{p-2}\left((p+1) \varepsilon+\frac{c_{2} T+\varepsilon}{\left\|x^{(j)}\right\|}+\frac{p c_{2} T+p c_{1}}{\left\|x^{(j)}\right\|^{2}}\right)
$$

for large $j$. Therefore, by $\varepsilon$ being chosen arbitrarily, there is a subsequence that converges to $y^{0} \in E_{T}$ such that

$$
\left\|\Delta y^{0}\right\|^{2} \leq a, \quad\left\|y^{0}\right\|=1
$$

On the other hand, we have

$$
\begin{aligned}
J_{p}\left(x^{(j)}\right)-\frac{1}{2}\left\langle J_{p}^{\prime}\left(x^{(j)}\right), x^{(j)}\right\rangle \\
=\left(\frac{1}{2}-\frac{1}{p}\right) \sum_{n=1}^{T} b(n)\left|x_{n}^{(j)}\right|^{p}-\sum_{n=1}^{T} G_{p}\left(n, x_{n}^{(j)}\right) \\
\quad+\frac{1}{2} \sum_{n=1}^{T}\left(g_{p}\left(n, x_{n}^{(j)}\right), x_{n}^{(j)}\right) .
\end{aligned}
$$

Then, by (9) and (11), for large $j$, we get

$$
\begin{aligned}
\left.\left|\left(\frac{1}{2}-\frac{1}{p}\right) \sum_{n=1}^{T} b(n)\right| x_{n}^{(j)}\right|^{p} \mid \\
=\mid J_{p}\left(x^{(j)}\right)-\frac{1}{2}\left\langle J_{p}^{\prime}\left(x^{(j)}\right), x^{(j)}\right\rangle+\sum_{n=1}^{T} G_{p}\left(n, x_{n}^{(j)}\right) \\
\quad-\frac{1}{2} \sum_{n=1}^{T}\left(g_{p}\left(n, x_{n}^{(j)}\right), x_{n}^{(j)}\right) \mid \\
\leq c_{1}+\frac{\varepsilon}{2}\left\|x^{(j)}\right\|+\varepsilon\left\|x^{(j)}\right\|^{2}+c_{2} T+\frac{1}{2}\left(\varepsilon\left\|x^{(j)}\right\|^{2}+c_{2} T\left\|x^{(j)}\right\|\right) .
\end{aligned}
$$

By dividing by $\left\|x^{(j)}\right\|^{p}$ in the previous formula, then by $p>2$, we have $\sum_{n=1}^{T} b(n)\left|y_{n}^{(j)}\right|^{p} \rightarrow 0$ as $j \rightarrow \infty$, that is, $\sum_{n=1}^{T} b(n)\left|y_{n}^{0}\right|^{p}=\lim _{j \rightarrow \infty} \sum_{n=1}^{T} b(n)\left|y_{n}^{(j)}\right|^{p}=0$. By the definition of $\lambda_{*}(p)$, see (6), we have $\left\|\Delta y^{0}\right\|^{2} \geq \lambda_{*}(p)$. This contradicts with (16) and assumption $a<\lambda_{*}(p)$. The proof is completed.

Lemma 5. Functional $J_{q}$ satisfies (PS) condition if $a<\lambda_{*}(q)$.

The proof is similar to that of Lemma 4 and is omitted.

2.2. Eigenvalue Problem. Consider eigenvalue problem:

$$
-\Delta^{2} x_{n-1}=\lambda x_{n}, \quad x_{n+T}=x_{n}, \quad x_{n} \in \mathbb{R}^{N},
$$

that is, $x_{n+1}+(\lambda-2) x_{n}+x_{n-1}=0, x_{n+T}=x_{n}$. By the periodicity, the difference system has complexity solution $x_{n}=e^{i n \theta} c$ for $c \in \mathbb{C}^{\mathrm{N}}$, where $\theta=2 k \pi / T, k \in \mathbb{Z}$. Moreover, $\lambda=2-e^{-i \theta}-e^{i \theta}=$ $2(1-\cos \theta)=4 \sin ^{2}(k \pi / T)$. Let $\eta_{k}$ denote the real eigenvector corresponding to the eigenvalues $\lambda_{k}=4 \sin ^{2}(k \pi / T)$, where $k \in Z[0, r]$ and $r=[T / 2]$. Since $a=4 \sin ^{2}(m \pi / T)$ for some $m \in Z[0, r]$, we can split space $E_{T}$ as follows:

$$
E_{T}=W^{-} \bigoplus W^{0} \bigoplus W^{+},
$$

where

$$
\begin{gathered}
W^{-}=\operatorname{span}\left\{\eta_{k} \mid k \in Z[0, m-1]\right\}, \quad W^{0}=\operatorname{span}\left\{\eta_{m}\right\}, \\
W^{+}=\operatorname{span}\left\{\eta_{k} \mid k \in Z[m+1, r]\right\} .
\end{gathered}
$$

By means of eigenvalue problem, we have $\left|\Delta x_{n}\right|^{2}-a\left|x_{n}\right|^{2}=$ $\left(\Delta x_{n}, \Delta x_{n}\right)-a\left(x_{n}, x_{n}\right)=\left(-\Delta^{2} x_{n-1}, x_{n}\right)-a\left(x_{n}, x_{n}\right)=(\lambda-$ a) $\left(x_{n}, x_{n}\right)=(\lambda-a)\left|x_{n}\right|^{2}$. Let

$$
\delta=\left\{\begin{array}{cl}
\min \left\{4 \sin ^{2} \frac{(m+1) \pi}{T}-4 \sin ^{2} \frac{m \pi}{T},\right. & \\
\left.4 \sin ^{2} \frac{m \pi}{T}-4 \sin ^{2} \frac{(m-1) \pi}{T}\right\}, & m \in Z[1, r], \\
4 \sin ^{2} \frac{\pi}{T}, & m=0 .
\end{array}\right.
$$

Then $\pm\left(\|\Delta x\|^{2}-a\|x\|^{2}\right) \geq \delta\|x\|^{2}$ for $x \in W^{ \pm}$. 
On the other hand, associating to numbers $\lambda_{*}(p)$ and $\lambda_{*}(q)$ (see (6)), we set

$$
\begin{aligned}
& \Lambda_{*}(p)=\sum_{n=1}^{T} b(n)\left|e_{n}\right|^{p}, \\
& \Lambda_{*}(q)=\sum_{n=1}^{T} b(n)\left|e_{n}\right|^{q},
\end{aligned}
$$

where $e_{n}=u \in \mathbb{R}^{N}(n \in[1, T])$ is the real eigenvector corresponding to eigenvalue $\lambda_{0}=0 . e=\left(e_{1}^{T}, e_{2}^{T}, \ldots, e_{N}^{T}\right)^{T}=$ $\left(u^{T}, u^{T}, \ldots, u^{T}\right)^{T} \in E_{T}$, where $\bullet^{T}$ denotes the transpose of a vector or a matrix. Moreover, letting $|u|=T^{-1 / 2}$, we have $\|e\|=1,\|\Delta e\|=0$. Therefore, by definition of $\lambda_{*}(p)$, if $\Lambda_{*}(p)=0$ then $\lambda_{*}(p)=0$.

However, by assumption $\lambda_{*}(p)>a=4 \sin ^{2}(m \pi / T)$ for some $m \in Z[0, r]$, thus $\lambda_{*}(p)>0$. That is to say the equality $\Lambda_{*}(p)=0$ cannot hold. Therefore our discussion will be distinguished in two cases: $\Lambda_{*}(p)>0$ and $\Lambda_{*}(p)<0$.

2.3. Preliminaries. Let $X$ be a Hilbert space, and let $\varphi \in$ $C^{1}(X, \mathbb{R})$ be a functional satisfying the (PS) condition. Write $\operatorname{crit}(\varphi)=\left\{x \in X \mid \varphi^{\prime}(x)=0\right\}$ for the set of critical points of functional $\varphi$ and $\varphi^{c}=\{x \in X \mid \varphi(x) \leq c\}$ for the level set. Denote by $H_{k}(A, B)$ the $k$ th singular relative homology group with integer coefficients. Let $x_{0} \in \operatorname{crit}(\varphi)$ be an isolated critical point with value $c=\varphi\left(x_{0}\right), c \in \mathbb{R}$, the group $C_{k}\left(\varphi, x_{0}\right)=H_{k}\left(\varphi^{c} \cap U,\left(\varphi^{c} \cap U\right) \backslash\left\{x_{0}\right\}\right)$, and $k \in \mathbb{Z}$ is called the $k$ th critical group of $\varphi$ at $x_{0}$, where $U$ is a closed neighbourhood of $u$. Due to the excision of homology [13], $C_{k}\left(\varphi, x_{0}\right)$ is dependent on $U$.

Suppose that $\varphi(\operatorname{crit}(\varphi))$ is strictly bounded from below by $a \in \mathbb{R}$, then the critical groups of $\varphi$ at infinity are formally defined [11] as $C_{k}(\varphi, \infty)=H_{k}\left(X, \varphi^{a}\right), k \in \mathbb{Z}$.

Proposition 6 (Proposition 2.3, [11]). Assume that $C^{2}$ functional $\varphi$ satisfying (PS) condition has a local linking at 0 with respect to $X=X_{0}^{+} \bigoplus X_{0}^{-}$; that is, there exists $\rho>0$ such that

$$
\begin{gathered}
\varphi(x) \leq \varphi(0) \quad \text { for } x \in X_{0}^{-} \text {and }\|x\| \leq \rho, \\
\varphi(x)>\varphi(0) \quad \text { for } x \in X_{0}^{+} \text {and } 0<\|x\| \leq \rho .
\end{gathered}
$$

Then $C_{k}(\varphi, 0) \neq 0, k=\operatorname{dim} X_{0}^{-}$.

By Propostion 6, one proves the following lemmas with respect to $E_{T}=X^{+} \bigoplus \mathrm{X}^{-}$.

Lemma 7. If $a<\lambda_{*}(p)$, then $C_{k}\left(J_{p}, 0\right) \neq 0, k=\operatorname{dim} X^{-}$, where $X^{-}=W^{-} \bigoplus W^{0}$ as $\Lambda_{*}(p)>0, X^{-}=W^{-}$as $\Lambda_{*}(p)<$ $0 . \Lambda_{*}(p)$ is defined by (23).

Proof. We first consider the following.

Case $1\left(\Lambda_{*}(p)>0\right.$ and $\left.X^{+}=W^{+}, X^{-}=W^{-} \bigoplus W^{0}\right)$. By $p>2,|x|^{p}=o\left(|x|^{2}\right)$ as $|x| \rightarrow 0$, then there exists $\theta \in(0,1)$ suitably small, such that $|x|^{p} \leq \delta / 3(b / p+\varepsilon)|x|^{2}$ as $|x|<\theta$, where $\delta>0$ see $(22)$ and $b=\max \{|b(1)|, \ldots,|b(T)|\}>0$. By assumption (A2) and $G_{p}(n, 0)=0$, for any given $\varepsilon>0$, there exists $\rho_{n} \in(0, \theta)$, such that $\left|G_{p}\left(n, x_{n}\right)\right| \leq \varepsilon\left|x_{n}\right|^{p}$ as $\left|x_{n}\right| \leq \rho_{n}$, $n \in Z[1, T]$. Thus

$$
\begin{aligned}
& \frac{1}{p} \sum_{n=1}^{T} b(n)\left|x_{n}\right|^{p}+\sum_{n=1}^{T} G_{p}\left(n, x_{n}\right) \\
& \quad \leq\left(\frac{b}{p}+\varepsilon\right) \sum_{n=1}^{T}\left|x_{n}\right|^{p} \leq \frac{1}{3} \delta\|x\|^{2} .
\end{aligned}
$$

Let $\rho=\min \left\{\rho_{1}, \ldots, \rho_{T}\right\}$. For $0<\|x\| \leq \rho<1$, it follows that

$$
J_{p}(x) \geq \frac{1}{2} \delta\|x\|^{2}-\frac{1}{3} \delta\|x\|^{2}>0, \quad x \in W^{+}=X^{+} .
$$

We need to prove that $J_{p}(x) \leq 0$ for $x \in X^{-}=W^{-} \bigoplus W^{0}$, $\|x\| \leq \rho$. We first claim that

$$
\sum_{n=1}^{T} b(n)\left|x_{n}\right|^{p}>0, \quad \forall x \in W^{-} \bigoplus W^{0}, x \neq 0 .
$$

Indeed, by contradiction, assume that $\sum_{n=1}^{T} b(n)\left|x_{n}\right|^{p} \leq 0$, for some $x \in W^{-} \bigoplus W^{0}, x \neq 0$. Since $\Lambda_{*}(p)=\sum_{n=1}^{T} b(n)\left|e_{n}\right|^{p}>$ 0 , where $e=\left(e_{1}^{T}, e_{2}^{T}, \ldots, e_{N}^{T}\right)^{T}=\left(u^{T}, u^{T}, \ldots, u^{T}\right)^{T} \in$ $W^{-} \oplus W^{0}$, and $\left(W^{-} \oplus W^{0}\right) \backslash\{0\}$ is arcwise connected, then there exists a $x^{0} \in\left(W^{-} \bigoplus W^{0}\right) \backslash\{0\}$, such that $\sum_{n=1}^{T} b(n)\left|x_{n}^{0}\right|^{p}=0$. Thus $\left\|\Delta x^{0}\right\|^{2} \geq \lambda_{*}(p)\left\|x^{0}\right\|^{2}$ by the definition of $\lambda_{*}(p)$. On the other hand, by the definition of $W^{-} \bigoplus W^{0}$, we have $\left\|\Delta x^{0}\right\|^{2} \leq a\left\|x^{0}\right\|^{2}$. This is a contradiction with assumption $a<\lambda_{*}(p)$. So the claim (27) holds.

There exists $c_{4}>0$ by (27), such that $\sum_{n=1}^{T} b(n)\left|x_{n}\right|^{p} \geq$ $c_{4}\|x\|_{p}^{p}$ for all $x \in W^{-} \bigoplus W^{0} \backslash\{0\}$, where $\|x\|_{p}=$ $\left(\sum_{n=1}^{T}\left|x_{n}\right|^{p}\right)^{1 / p}$. For $x \in W^{-} \bigoplus W^{0},\|x\| \leq \rho, \varepsilon$ sufficiently small, we have

$$
\begin{aligned}
J_{p}(x) & \leq-\frac{1}{p} \sum_{n=1}^{T} b(n)\left|x_{n}\right|^{p}-\sum_{n=1}^{T} G_{p}\left(n, x_{n}\right) \\
& \leq-\frac{c_{4}}{p}\|x\|_{p}^{p}+\varepsilon\|x\|_{p}^{p} \leq 0 .
\end{aligned}
$$

Since $J_{p}(0)=0$ and $J_{p}$ satisfies (PS) condition by Lemma 4 , so by Proposition 6, we obtain that $C_{k}\left(J_{p}, 0\right) \neq 0$ for $k=$ $\operatorname{dim}\left(W^{-} \oplus W^{0}\right)$.

Case $2\left(\Lambda_{*}(p)<0, X^{+}=W^{+} \bigoplus W^{0}, X^{-}=W^{-}\right)$. It is easy to see that $J_{p}(x) \leq 0$ by $\|\Delta x\|^{2}-a\|x\|^{2} \leq-\delta\|x\|^{2}$ and $p>2$, where $x \in W^{-}$and $\|x\| \leq \rho$. We need to claim that $J_{p}(x)>0$, for $x \in W^{+} \bigoplus W^{0}, 0<\|x\| \leq \rho$.

Suppose not that there exists a sequence $\left\{x^{(j)}\right\} \subset E_{T}$ such that

$$
\begin{gathered}
\left\{x^{(j)}\right\} \subset W^{+} \bigoplus W^{0} \backslash\{0\}, \quad 0<\left\|x^{(j)}\right\| \leq \rho, \\
J_{p}\left(x^{(j)}\right) \leq 0,
\end{gathered}
$$


for large $j$. For $\left\|x^{(j)}\right\| \leq \rho$, by Lemma 1, we get

$$
\begin{aligned}
& \left|\sum_{n=1}^{T}\left[\frac{1}{p} b(n)\left|x_{n}^{(j)}\right|^{p}+G_{p}\left(n, x_{n}^{(j)}\right)\right]\right| \\
& \quad \leq \sum_{n=1}^{T}\left[\frac{b}{p}\left|x_{n}^{(j)}\right|^{p}+\varepsilon\left|x_{n}^{(j)}\right|^{p}\right] \leq\left(\frac{b}{p}+\varepsilon\right)\left(\frac{1}{a_{1}}\right)^{p}\left\|x^{(j)}\right\|^{p} .
\end{aligned}
$$

Set $y_{n}^{(j)}=x_{n}^{(j)} /\left\|x^{(j)}\right\|$. Then by (29) and the previous formula, we have

$$
\begin{aligned}
0 \geq \frac{J_{p}\left(x^{(j)}\right)}{\left\|x^{(j)}\right\|^{2}} \geq & \frac{1}{2}\left(\left\|\Delta y^{(j)}\right\|^{2}-a\right) \\
& -\left(\frac{b}{p}+\varepsilon\right)\left(\frac{1}{a_{1}}\right)^{p}\left\|x^{(j)}\right\|^{p-2} .
\end{aligned}
$$

On the other hand, $\left\|\Delta y^{(j)}\right\|^{2} \geq a$ by the definition of $W^{+} \oplus W^{0}$. Hence by $p>2$, there exists a subsequence converges to $y^{0} \in E_{T}$, such that $\left\|\Delta y^{0}\right\|^{2}=a$, that is $y^{0} \in$ $W^{0}$ and $\left\|y^{0}\right\|=1$. Since $\left\|\Delta x^{(j)}\right\|^{2} \geq a\left\|x^{(j)}\right\|^{2}$ for $\left\{x^{(j)}\right\} \subset$ $W^{+} \bigoplus W^{0}$, it follows from $J_{p}\left(x^{(j)}\right) \leq 0$ that

$$
\begin{aligned}
0 & \leq \frac{1}{p} \sum_{n=1}^{T} b(n)\left|x_{n}^{(j)}\right|^{p}+\sum_{n=1}^{T} G_{p}\left(n, x_{n}^{(j)}\right) \\
& \leq \frac{1}{p} \sum_{n=1}^{T} b(n)\left|x_{n}^{(j)}\right|^{p}+\varepsilon\left(\frac{1}{a_{1}}\right)^{p}\left\|x^{(j)}\right\|^{p} .
\end{aligned}
$$

Dividing by $\left\|x^{(j)}\right\|^{p}$ in the previous inequality, then $\sum_{n=1}^{T} b(n)\left|y_{n}^{0}\right|^{p}=\lim _{j \rightarrow \infty} \sum_{n=1}^{T} b(n)\left|y_{n}^{(j)}\right|^{p} \geq 0$.

Since $e, y^{0} \in W^{-} \bigoplus W^{0}, \Lambda_{*}(p)=\sum_{n=1}^{T} b(n)\left|e_{n}\right|^{p}<0$ and $\left(W^{-} \bigoplus W^{0}\right) \backslash\{0\}$ is arcwise connected, then there exists a $\bar{y} \in\left(W^{-} \bigoplus W^{0}\right) \backslash\{0\}$ such that $\sum_{n=1}^{T} b(n)\left|\bar{y}_{n}\right|^{p}=0$. Thus $\|\Delta \bar{x}\|^{2} \geq \lambda_{*}(p)\|\bar{x}\|^{2}$ by the definition of $\lambda_{*}(p)$. On the other hand, $\|\Delta \bar{x}\|^{2} \leq a\|\bar{x}\|^{2}$ by the definition of $W^{-} \bigoplus W^{0}$. This is a contradiction with assumption $a<\lambda_{*}(p)$. That is to say, the claim is valid.

By Proposition 6, we obtain $C_{k}\left(J_{p}, 0\right) \neq 0, k=\operatorname{dim} W^{-}$. The proof is completed.

Lemma 8. If $a<\lambda_{*}(q)$, then $C_{k}\left(J_{q}, \infty\right) \neq 0$ for $k=\operatorname{dim} X^{-}$, where $X^{-}=W^{-} \bigoplus W^{0}$ as $\Lambda_{*}(q)>0, X^{-}=W^{-}$as $\Lambda_{*}(q)<0$.

The proof is similar to that of Lemma 7 and is omitted.

\section{Proof of Theorem 2}

Lemma 9. Let $a<\lambda_{*}(p)$. If there exists $K_{1}>0$ such that for any $K>K_{1}, J_{p}(x) \leq-K$, then one has $\sum_{n=1}^{T} b(n)\left|x_{n}\right|^{p}>0$, and $\left.(d / d t) J_{p}(t x)\right|_{t=1}<0$.

Proof. We first claim that $\|x\|$ is sufficiently large, if $x$ satisfies condition of Lemma 9. Suppose not there exists $M>$ 0 such that $\|x\| \leq M$. So there exists $\left\{x^{(j)}\right\} \subset E_{T}, x^{0} \in E_{T}$, such that $x^{(j)} \rightarrow x^{0}$ as $j \rightarrow \infty$. Since for any $j>K_{1}$, we have $J_{p}\left(x^{(j)}\right) \leq-j$, thus $J_{p}\left(x^{0}\right)=\lim _{j \rightarrow \infty} J_{p}\left(x^{(j)}\right)=-\infty$. It is a contradiction with $J_{p}\left(x^{0}\right)=c$.

If $\|x\|$ is large enough, then we can assume that $\left|x_{n}\right|$ is large enough for $n \in Z[1, k]$ and $\left|x_{n}\right|$ are bounded for $n \in Z[k+$ $1, T]$. Therefore, by assumption (A1), for any given $\varepsilon>0$, there exists $M_{1}>0$ such that

$$
\begin{gathered}
\left|g_{p}\left(n, x_{n}\right)\right| \leq \varepsilon\left|x_{n}\right|+\frac{M_{1}}{T}, \quad\left|G_{p}\left(n, x_{n}\right)\right| \leq \varepsilon\left|x_{n}\right|^{2}+\frac{M_{1}}{T}, \\
\forall\left(n, x_{n}\right) \in Z[1, T] \times \mathbb{R}^{N} .
\end{gathered}
$$

We claim that $\sum_{n=1}^{T} b(n)\left|x_{n}\right|^{p}>0$. Suppose not that, for $j>$ $K_{1}$, there exists $\left\{x^{(j)}\right\} \subset E_{T}$ such that

$$
\sum_{n=1}^{T} b(n)\left|x_{n}^{(j)}\right|^{p} \leq 0 .
$$

By $J_{p}\left(x^{(j)}\right) \leq-j \leq 0,(33)$ and (34), we have

$$
\begin{gathered}
\frac{1}{2}\left\|\Delta x^{(j)}\right\|^{2} \leq \frac{a}{2}\left\|x^{(j)}\right\|^{2}+\sum_{n=1}^{T} G_{p}\left(n, x_{n}^{(j)}\right) \\
\leq \frac{a}{2}\left\|x^{(j)}\right\|^{2}+\varepsilon\left\|x^{(j)}\right\|^{2}+M_{1} .
\end{gathered}
$$

Set $y_{n}^{(j)}=x_{n}^{(j)} /\left\|x^{(j)}\right\|$ and divided by $\left\|x^{(j)}\right\|^{2}$ in the previous inequality. Since $\varepsilon$ can be small enough, then there exists a subsequence that converges to $y^{0} \in E_{T}$, such that $\left\|\Delta y^{0}\right\|^{2} \leq a$, $\left\|y^{0}\right\|=1$. Moreover, by (33) and (34), we get

$$
\begin{aligned}
0 \geq & \frac{1}{p} \sum_{n=1}^{T} b(n)\left|x_{n}^{(j)}\right|^{p} \geq j+\frac{1}{2}\left\|\Delta x^{(j)}\right\|^{2}-\frac{a}{2}\left\|x^{(j)}\right\|^{2} \\
& -\sum_{n=1}^{T} G_{p}\left(n, x_{n}^{(j)}\right) \geq-\left(\frac{a}{2}+\varepsilon\right)\left\|x^{(j)}\right\|^{2}-M_{1} .
\end{aligned}
$$

Since $p>2$ and $\lim _{j \rightarrow \infty}\left\|x^{(j)}\right\|=\infty$, divided by $\left\|x^{(j)}\right\|^{p}$ in the previous inequality, we have $\sum_{n=1}^{T} b(n)\left|y_{n}^{0}\right|^{p}=$ $\lim _{j \rightarrow \infty} \sum_{n=1}^{T} b(n)\left|y_{n}^{(j)}\right|^{p}=0$, that is, $\left\|\Delta y^{0}\right\| \geq \lambda_{*}(q)$, which deduce a contradiction. So the claim $\sum_{n=1}^{T} b(n)\left|x_{n}\right|^{p}>0$ holds.

Next we prove that $\left.(d / d t) J_{p}(t x)\right|_{t=1}<0$ holds. By contradiction, there exists a sequence $\left\{x^{(j)}\right\} \subset E_{T}$ such that, for $j>K_{1}$,

$$
\left.\frac{d}{d t} J_{p}\left(t x^{(j)}\right)\right|_{t=1} \geq 0 .
$$

Then, by (7), we get

$$
\begin{aligned}
\left.\frac{d}{d t} J_{p}\left(t x^{(j)}\right)\right|_{t=1}= & \left\|\Delta x^{(j)}\right\|^{2}-a\left\|x^{(j)}\right\|^{2} \\
& -\sum_{n=1}^{T} b(n)\left|x_{n}^{(j)}\right|^{p}-\sum_{n=1}^{T}\left(g_{p}\left(n, x_{n}^{(j)}\right), x_{n}^{(j)}\right),
\end{aligned}
$$


and by (37) and $J_{p}\left(x^{(j)}\right) \leq-j<0$, it follows that

$$
\begin{aligned}
\left(1-\frac{p}{2}\right) & \left(\left\|\Delta x^{(j)}\right\|^{2}-a\left\|x^{(j)}\right\|^{2}\right) \\
& -\sum_{n=1}^{T}\left(g_{p}\left(n, x_{n}^{(j)}\right), x_{n}^{(j)}\right)+p \sum_{n=1}^{T} G_{p}\left(n, x_{n}^{(j)}\right) \\
= & \left.\frac{d}{d t} J_{p}\left(t x^{(j)}\right)\right|_{t=1}-p J_{p}\left(x^{(j)}\right) \geq 0 .
\end{aligned}
$$

Set $y_{n}^{(j)}=x_{n}^{(j)} /\left\|x^{(j)}\right\|$ and divided by $\left\|x^{(j)}\right\|^{2}$ in the previous formula; since $p>2$ and $\varepsilon$ can be small enough, then there exists a subsequence converges to $y^{0} \in E_{T}$ such that $\left\|\Delta y^{0}\right\|^{2} \leq a,\left\|y^{0}\right\|=1$. Moreover, by (37) and the first claim, we get

$$
\begin{aligned}
0< & \sum_{n=1}^{T} b(n)\left|x_{n}^{(j)}\right|^{p} \leq\left\|\Delta x^{(j)}\right\|^{2}-a\left\|x^{(j)}\right\|^{2} \\
& -\sum_{n=1}^{T}\left(g_{p}\left(n, x_{n}^{(j)}\right), x_{n}^{(j)}\right) .
\end{aligned}
$$

Divided by $\left\|x^{(j)}\right\|^{p}$ in the previous formula, and by $p>2$, it follows that $\sum_{n=1}^{T} b(n)\left|y_{n}^{0}\right|^{p}=0$. This is a contradiction with the definition of $\lambda_{*}(p)$ and condition $a<\lambda_{*}(p)$. So the second claim holds. The proof is completed.

Based on Lemma 9, we introduce the following notations:

$$
\begin{gathered}
J_{p}^{-K}=\left\{x \in E_{T}: J_{p}(x) \leq-K\right\}, \\
E_{p}^{+}=\left\{x \in E_{T}: \sum_{n=1}^{T} b(n)\left|x_{n}\right|^{p}>0\right\}, \\
E\left(\Omega_{+}\right)=\left\{x \in E_{T}: x_{n}=0 \text { for } n \in Z[1, T] \backslash \Omega_{+}\right\} \backslash\{0\} .
\end{gathered}
$$

Clearly, $E\left(\Omega_{+}\right) \subset E_{p}^{+}$. And by Lemma 9, we have $J_{p}^{-K} \subset E_{p}^{+}$. In order to describe the $H_{q}\left(E_{T}, J_{p}^{-K}\right)$, we need to show the following lemma.

Lemma 10. If $a<\lambda_{*}(p)$, then there exists $K_{1}>0$, such that for any $K>K_{1}, J_{p}^{-K}$ is a strong deformation retraction of $E_{p}^{+}$. Moreover, $E\left(\Omega_{+}\right)$and $E_{p}^{+}$are homotopy equivalent.

Proof. Now we prove that $J_{p}^{-K}$ is a strong deformation retraction of $E_{p}^{+}$.

By Lemma 9, we have $J_{p}^{-K} \subset E_{p}^{+}$. Let $x \in E_{p}^{+}$. By Lemma 9, there exists a unique $t_{p}=t_{p}(x)>0$ such that $J_{p}\left(t_{p} x\right)=-K$. By applying Implicit Function Theorem, $t_{p}(x)$ is a continuous function in $E_{p}^{+}$. Let $T_{p}(x)=\max \left\{t_{p}(x), 1\right\}$ and define $f_{p}(s, x)=(1-s) x+s T_{p}(x) x$, then $f_{p}:[0,1] \times E_{p}^{+} \rightarrow$ $J_{p}^{-K}$ is a strong deformation retraction. Thus $J_{p}^{-K}$ is a strong deformation retraction of $E_{p}^{+}$.
We next claim that $E\left(\Omega_{+}\right)$is a strong deformation retraction of $E_{p}^{+}$. Clearly, in terms of the notations, we have $E\left(\Omega_{+}\right) \subset$ $E_{p}^{+}$. Let $\xi_{p}: Z[1, T] \rightarrow \mathbb{R}$ be a function such that

$$
\begin{array}{r}
\xi_{p}(n)=1 \quad \text { if } n \in \Omega_{+}, \quad \xi_{p}(n)=0 \quad \text { if } n \in \Omega_{-}, \\
\xi_{p}(n) \in[0,1] \quad \text { if } n \in Z[1, T] \backslash\left(\Omega_{+} \cup \Omega_{-}\right) .
\end{array}
$$

Define

$$
\zeta_{p}\left(s, x_{n}\right)=\left\{\begin{array}{lr}
(1-2 s) x_{n}+2 s \xi_{p}(n) x_{n} & \text { if } 0 \leq s \leq \frac{1}{2} \\
2(1-s) \xi_{p}(n) x_{n}+2\left(s-\frac{1}{2}\right) P\left(\xi_{p}(n) x_{n}\right) & \text { if } \frac{1}{2} \leq s \leq 1,
\end{array}\right.
$$

where $P: E_{T} \rightarrow E\left(\Omega_{+}\right)$is a projection operator. Then $\zeta_{p}$ : $[0,1] \times E_{p}^{+} \rightarrow E\left(\Omega_{+}\right)$is a deformation retraction. Indeed,

$$
\begin{gathered}
\zeta_{p}(0, x)=x, \quad \zeta_{p}(1, x) \in E\left(\Omega_{+}\right), \quad \text { for } x \in E_{p}^{+}, \\
\zeta_{p}(s, x)=x, \quad \text { for } x \in E\left(\Omega_{+}\right) \text {and } s \in[0,1] .
\end{gathered}
$$

For $x \in E_{p}^{+}$, if $s \in[0,1 / 2]$, then

$$
\begin{aligned}
\sum_{n=1}^{T} b(n)\left|\zeta_{p}\left(s, x_{n}\right)\right|^{p} & \\
& =\sum_{n \in \Omega_{+}} b(n)\left|x_{n}\right|^{p}+\sum_{n \in \Omega_{-}} b(n)(1-2 s)^{p}\left|x_{n}\right|^{p} \\
& \geq \sum_{n=1}^{T} b(n)\left|x_{n}\right|^{p}>0,
\end{aligned}
$$

where $0 \leq(1-2 s)^{p} \leq 1$, that is, $\zeta_{p}(s, x) \in E_{p}^{+}$. If $s \in(1 / 2,1]$, it follows that

$$
\begin{aligned}
& \sum_{n=1}^{T} b(n)\left|\zeta_{p}\left(s, x_{n}\right)\right|^{p} \\
& \quad=\sum_{n \in \Omega_{+}} b(n)\left|2(1-s) \xi_{p}(n) x_{n}+2\left(s-\frac{1}{2}\right) P\left(\xi_{p}(n) x_{n}\right)\right|^{p} \\
& \quad \geq 0 .
\end{aligned}
$$

We claim that the equality of the previous formula cannot hold. Otherwise, $P x_{n}=-((1-s) /(s-(1 / 2))) x_{n}$, for $n \in \Omega_{+}$, which implies that $P x_{n}=0$. Hence $x_{n}=0$ in $\Omega_{+}$, which contradicts with the fact $x \in E_{p}^{+}$. So $\sum_{n=1}^{T} b(n)\left|\zeta_{p}\left(s, x_{n}\right)\right|^{p}>$ 0 , that is, $\zeta_{p}(s, x) \in E_{p}^{+}$as $s \in(1 / 2,1]$. Therefore, $\zeta_{p}$ is a deformation retraction from $E_{p}^{+}$onto $E\left(\Omega_{+}\right)$, and this completes the proof.

Proof of Theorem 2. Since $E\left(\Omega_{+}\right)$is well known to be contractile in itself, and by Lemma 10 , it follows that $J_{p}^{-K}$ is 
homotopically equivalent to $E\left(\Omega_{+}\right)$for $K$ large enough, then the Betti numbers (cf. $[11,13])$ are

$$
\begin{aligned}
\beta_{k} & =\operatorname{dim} C_{k}\left(J_{p}, \infty\right)=\operatorname{dim} H_{k}\left(E_{T}, J_{p}^{-K}\right) \\
& =\operatorname{dim} H_{k}\left(E_{T}, E\left(\Omega_{+}\right)\right)=0, \quad k \in Z[0, N T] .
\end{aligned}
$$

Now we suppose that system (4) has only trivial solution; that is, $J_{p}$ has only critical point $x=0$, then we have the Morse-type numbers $M_{k}=\operatorname{dim} C_{k}\left(J_{p}, 0\right)$ for $k \epsilon$ $Z[0, N T]$ (cf. [13]). Moreover, by Lemma 7, $C_{k}\left(J_{p}, 0\right) \neq 0$ for $k=\operatorname{dim} W^{-}$or $k=\operatorname{dim}\left(W^{-} \bigoplus W^{0}\right)$. Since $J_{p}$ satisfies (PS) condition by Lemma 4, then using Morse Relation, we have the following.

$$
0=\sum_{k=0}^{N T}(-1)^{k} \beta_{k}=\sum_{k=0}^{N T}(-1)^{k} M_{k} \neq 0,
$$

which is a contradiction. Therefore, $J_{p}$ has at least one critical point $x^{*} \neq 0$ and system (4) has at least a nonzero $T$-periodic solution.

\section{Proof of Theorem 3}

For convenience, we introduce the following notations:

$$
\begin{aligned}
& J_{q}^{c}=\left\{x \in E_{T}: J_{q}(x) \leq c\right\}, \quad c \in \mathbb{R}, \\
& E_{q}^{+}=\left\{x \in E_{T}: \sum_{n=1}^{T} b(n)\left|x_{n}\right|^{q}>0\right\} .
\end{aligned}
$$

Clearly, $E_{q}^{+} \cup\{0\}$ is star-shaped with respect to the origin and $E\left(\Omega_{+}\right) \subset E_{q}^{+}$, where $E\left(\Omega_{+}\right)$is given in Section 3. Similarly with the proof of Lemmas 9 and 10, we have the following.

Lemma 11. Let $a<\lambda_{*}(q)$. Then there exists $\rho>0$ such that $\left.(d / d t) J_{q}(t x)\right|_{t=1}>0$ for any $x \in M_{\rho}=\left\{x \in B_{\rho} \cap E_{q}^{+}: J_{q}(x) \geq\right.$ $0\}$, where $B_{\rho}$ stands for the closed ball in $E_{T}$ of radius $\rho>0$ with the center at zero.

Lemma 12. Let $a<\lambda_{*}(q)$. Then there exists $\rho>0$ such that $\left(J_{q}^{0} \cap B_{\rho}\right) \backslash\{0\}$ is a retract of $E_{q}^{+} \cap B_{\rho}$, and $E\left(\Omega^{+}\right)$is a strong deformation retraction of $E_{q}^{+}$.

Proof of Theorem 3. We first prove that $J_{q}^{0} \cap B_{\rho}$ is contractible in itself. In fact, it is sufficient to show that $J_{q}^{0} \cap B_{\rho}$ is starshaped with respect to the origin; that is, $x \in J_{q}^{0} \cap B_{\rho}$ implies that $t x \in J_{q}^{0} \cap B_{\rho}$ for all $t \in[0,1]$.

Assume, by a contradiction, that there exists $x_{0} \in J_{q}^{0} \cap B_{\rho}$ and $t_{0} \in(0,1)$, such that $J_{q}\left(t_{0} x_{0}\right)>0$. It follows from Lemma 11 that $(d / d t) J_{q}\left(t_{0} x_{0}\right)>0$. By the monotonicity arguments, this implies that

$$
J_{q}\left(t x_{0}\right)>0 \quad \forall t \in\left[t_{0}, 1\right] .
$$

This contradicts the assumption $x_{0} \in J_{q}^{0}$, which implies $J_{q}\left(x_{0}\right) \leq 0$.
On the other hand, since $E\left(\Omega_{+}\right)$is contractible in itself, and $E_{q}^{+} \cup\{0\}$ is starshaped with respect to the origin, then $E_{q}^{+} \cap B_{\rho}$ is contractible in itself. The retract of the set which is contractible in itself is also contractible (cf. [19]); it follows that the set $\left(J_{q}^{0} \cap B_{\rho}\right) \backslash\{0\}$ is contractible by Lemma 12 .

Combining the previous argument, $J_{q}^{0} \cap B_{\rho}$ and $\left(J_{q}^{0} \cap B_{\rho}\right) \backslash$ $\{0\}$ are contractible in themselves.

$$
\begin{array}{r}
\operatorname{dim} C_{k}\left(J_{q}, 0\right)=\operatorname{dim} H_{k}\left(J_{q}^{0} \cap B_{\rho},\left(J_{q}^{0} \cap B_{\rho}\right) \backslash\{0\}\right)=0, \\
k \in Z[0, N T] .
\end{array}
$$

By Lemma $8, C_{k}\left(J_{q}, \infty\right) \neq 0$ for $k=\operatorname{dim}\left(W^{-} \bigoplus W^{0}\right)$ or $k=$ $\operatorname{dim} W^{-}$. Therefore, by Morse Relation and the same methods in proof of Theorem 2, it follows that $J_{q}$ has at least one critical point $x^{*} \neq 0$ and system (5) has at least a nonzero $T$-periodic solution.

\section{Acknowledgments}

This research is supported by the National Natural Science Foundation of China under Grants (11101187) and NCETFJ (JA11144), the Excellent Youth Foundation of Fujian Province (2012J06001), and the Foundation of Education of Fujian Province (JA09152).

\section{References}

[1] Z. Guo and J. Yu, "Existence of periodic and subharmonic solutions for second-order superlinear difference equations," Science in China A, vol. 46, no. 4, pp. 506-515, 2003.

[2] Z. Guo and J. Yu, "The existence of periodic and subharmonic solutions of subquadratic second order difference equations," Journal of the London Mathematical Society, vol. 68, no. 2, pp. 419-430, 2003.

[3] W. G. Kelley and A. C. Peterson, Difference Equations: An Introduction with Applications, Academic Press, Boston, Mass, USA, 1991.

[4] C.-L. Tang and X.-P. Wu, "Periodic solutions for second order Hamiltonian systems with a change sign potential," Journal of Mathematical Analysis and Applications, vol. 292, no. 2, pp. 506516, 2004.

[5] Y.-T. Xu and Z.-M. Guo, "Existence of periodic solutions to second-order Hamiltonian systems with potential indefinite in sign," Nonlinear Analysis. Theory, Methods \& Applications A, vol. 51, no. 7, pp. 1273-1283, 2002.

[6] C. D. Ahlbrandt and A. C. Peterson, Discrete Hamiltonian Systems: Difference Equations, Continued Fractions, and Riccati Equations, vol. 16, Kluwer Academic Publishers, Dordrecht, The Netherlands, 1996.

[7] H. Amann and E. Zehnder, "Nontrivial solutions for a class of nonresonance problems and applications to nonlinear differential equations," Annali della Scuola Normale Superiore di Pisa. Classe di Scienze. Serie IV, vol. 7, no. 4, pp. 539-603, 1980.

[8] Z. Huang, C. Feng, and S. Mohamad, "Multistability analysis for a general class of delayed Cohen-Grossberg neural networks," Information Sciences, vol. 187, pp. 233-244, 2012. 
[9] Z. Huang and Y. N. Raffoul, "Biperiodicity in neutral-type delayed difference neural networks," Advances in Difference Equations, vol. 2012, article 5, 2012.

[10] W. Zou and S. Li, "Infinitely many solutions for Hamiltonian systems," Journal of Differential Equations, vol. 186, no. 1, pp. 141164, 2002.

[11] T. Bartsch and S. Li, "Critical point theory for asymptotically quadratic functionals and applications to problems with resonance," Nonlinear Analysis. Theory, Methods \& Applications A, vol. 28, no. 3, pp. 419-441, 1997.

[12] K. C. Chang, Critical Point Theory and Its Applications, Science and Technical Press, Shanghai, China, 1980.

[13] J. Mawhin and M. Willem, Critical Point Theory and Hamiltonian Systems, vol. 74, Springer, New York, NY, USA, 1989.

[14] P. H. Rabinowitz, Minimax Methods in Critical Point Theory with Applications to Differential Equations, vol. 65 of CBMS Regional Conference Series in Mathematics, American Mathematical Society, Providence, RI, USA, 1986.

[15] V. Benci, "A new approach to the Morse-Conley theory and some applications," Annali di Matematica Pura ed Applicata, vol. 158, pp. 231-305, 1991.

[16] C. Conley, Isolated Invariant Sets and the Morse Index, vol. 38 of CBMS Regional Conference Series in Mathematics, American Mathematical Society, Providence, RI, USA, 1978.

[17] K. C. Chang, "Solutions of asymptotically linear operator equations via Morse theory," Communications on Pure and Applied Mathematics, vol. 34, no. 5, pp. 693-712, 1981.

[18] K.-C. Chang, Infinite-Dimensional Morse Theory and Multiple Solution Problems, Birkhäuser, Boston, Mass, USA, 1993.

[19] V. Moroz, "On the Morse critical groups for indefinite sublinear elliptic problems," Nonlinear Analysis. Theory, Methods \& Applications A, vol. 52, no. 5, pp. 1441-1453, 2003.

[20] F. Antonacci, "Periodic and homoclinic solutions to a class of Hamiltonian systems with indefinite potential in sign," Unione Matematica Italiana. Bollettino B, vol. 10, no. 2, pp. 303-324, 1996.

[21] F. Antonacci, "Existence of periodic solutions of Hamiltonian systems with potential indefinite in sign," Nonlinear Analysis. Theory, Methods \& Applications A, vol. 29, no. 12, pp. 1353-1364, 1997.

[22] H.-H. Bin, J.-S. Yu, and Z.-M. Guo, "Nontrivial periodic solutions for asymptotically linear resonant difference problem," Journal of Mathematical Analysis and Applications, vol. 322, no. 1, pp. 477-488, 2006. 


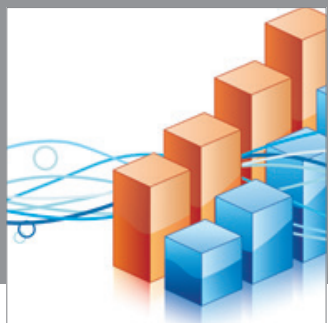

Advances in

Operations Research

mansans

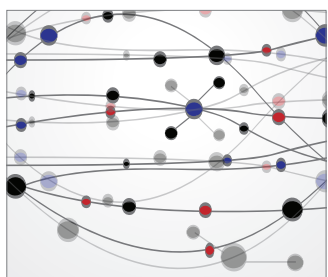

The Scientific World Journal
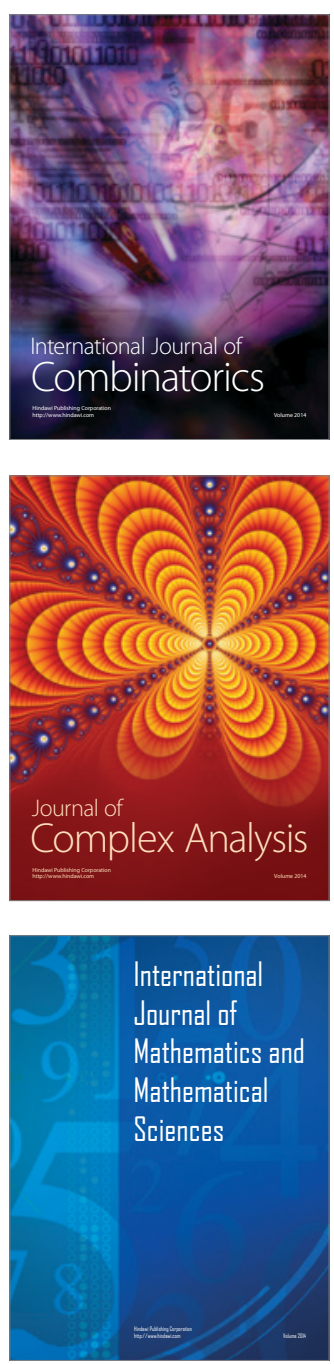
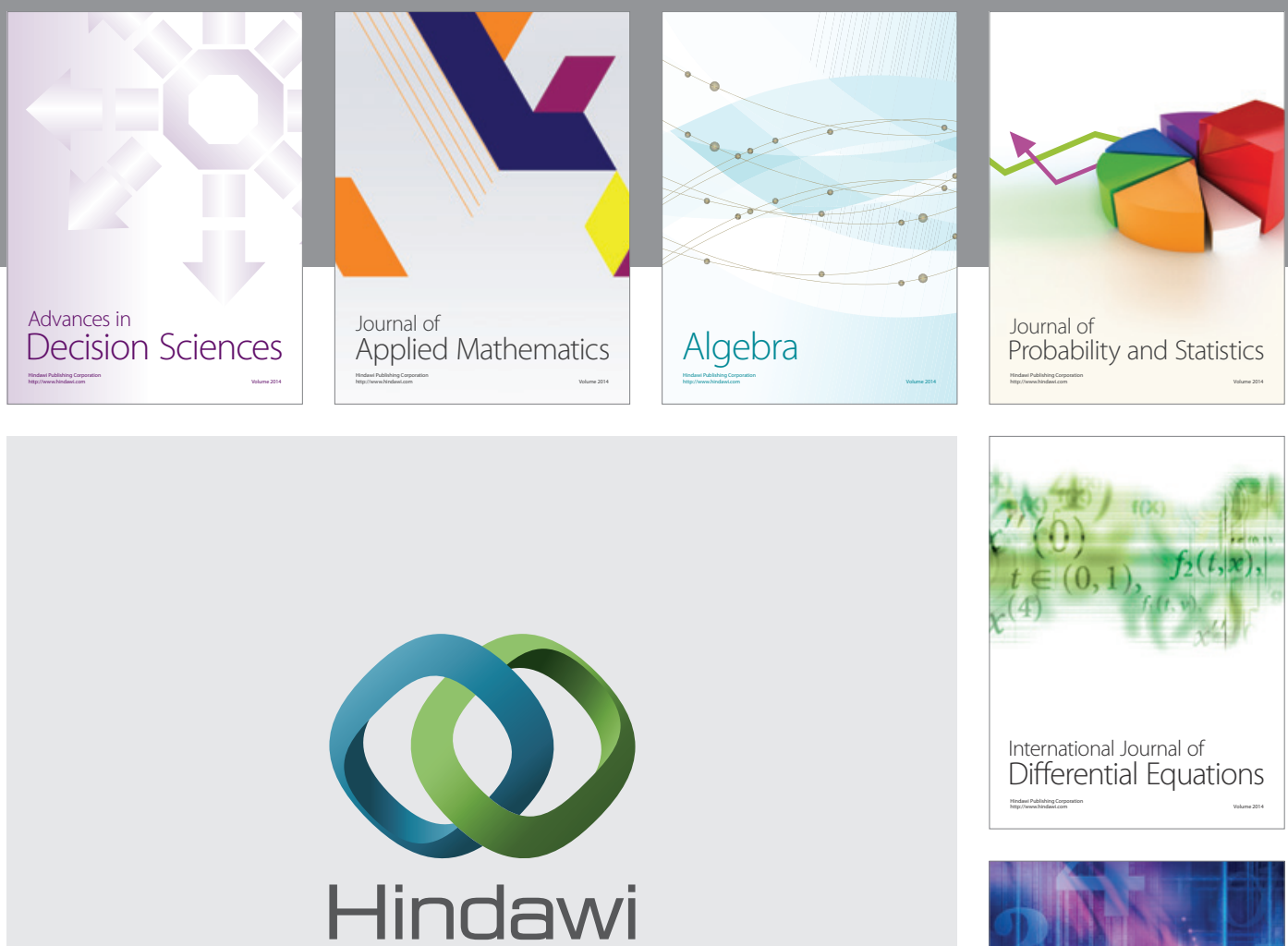

Submit your manuscripts at http://www.hindawi.com
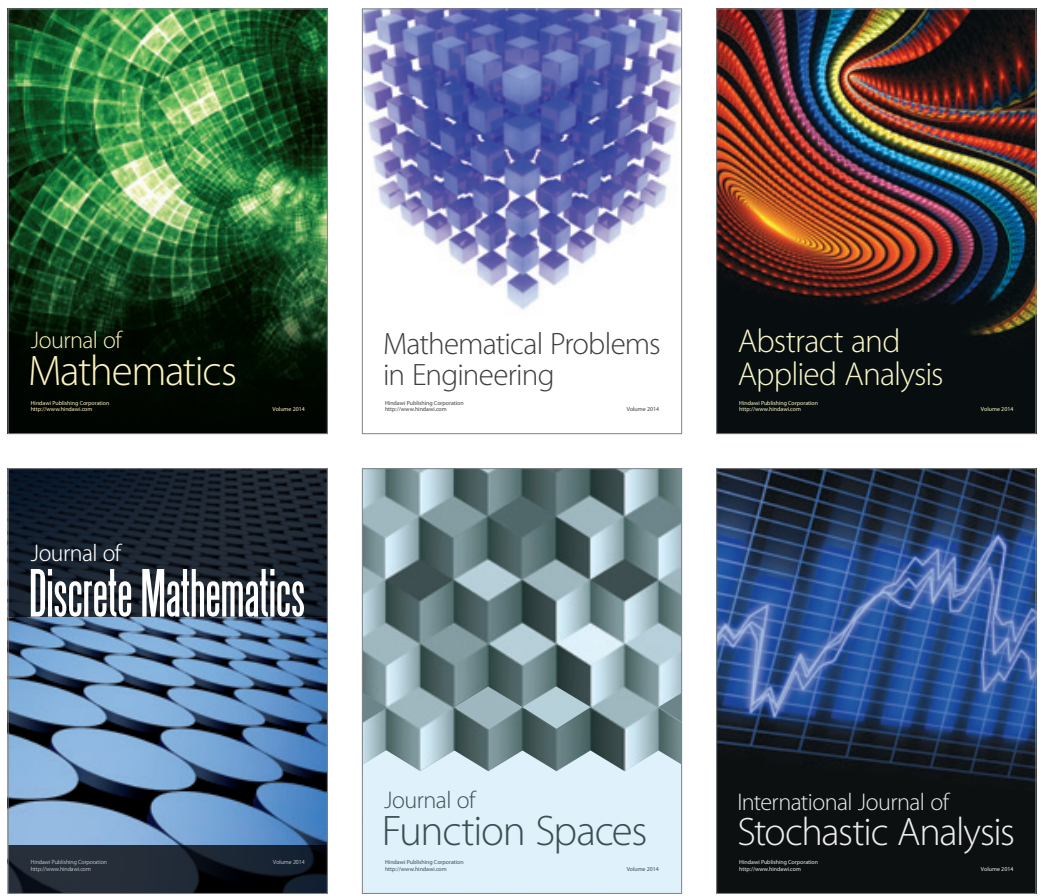

Journal of

Function Spaces

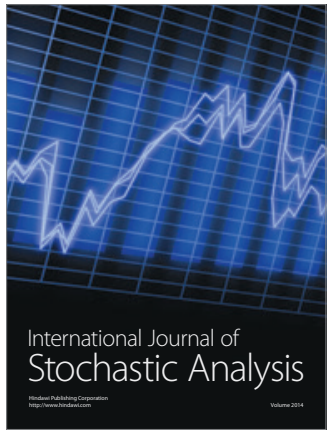

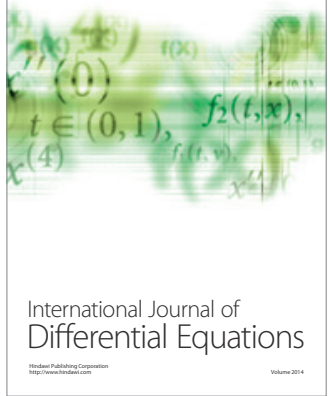
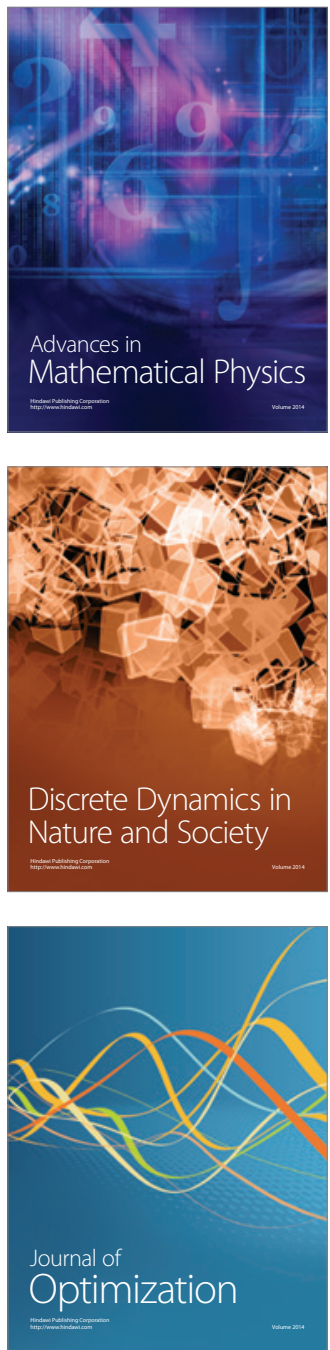